Ann. Génét. Sél. anim., I974, 6 (I), Iо3-II6.

\title{
DÉTERMINISME DE LA DURÉE DE DÉVELOPPEMENT CHEZ DROSOPHILA MELANOGASTER
}

\author{
F. A. LINTS et G. GRUWEZ \\ Laboratoire de Génétique \\ Faculté des Sciences agronomiques \\ Université de Louvain \\ Kardinaal Mercierlaan, 92 \\ 3030 Heverlee (Belgique)
}

\begin{abstract}
RÉSUMÉ
Chez Drosophila melanogaster, deux expériences de sélection deux-voies, l'une pour la taille, l'autre pour la durée de développement, ont été conçues de telle sorte qu'il ne soit procédé à aucune sélection inconsciente d'un caractère par l'intermédiaire d'une sélection incontrôlée de l'autre. La taille et la durée de développement furent mesurées dans les deux expériences. Tous les facteurs d'environnement, dont l'influence sur la durée de développement et la taille est connue, furent contrôlés et maintenus constants autant qu'il est possible. Il est montré que la sélection pour la durée de développement, lorsqu'elle n'est pas influencée par une sélection pour un autre caractère, est inefficace; que des fluctuations importantes de la durée de développement s'observent de génération en génération; que ces fluctuations ne dépendent ni du génotype ni de l'environnement mais sont, pour une génération donnée, déterminées par la durée de développement de la génération précédente. Les résultats sont discutés en termes de dimension temporelle de la morphogenèse.
\end{abstract}

\section{INTRODUCTION}

Classiquement, on considère que le phénotype d'un organisme est la résultante de l'interaction des gènes de cet organisme avec le milieu dans lequel il vit. Les gènes impliqués sont de deux ordres : les gènes majeurs déterminent les caractères discontinus ou qualitatifs, les gènes mineurs ou polygènes les caractères continus ou quantitatifs. L'activité des polygènes peut être de nature additive ou non-additive. Diverses méthodes statistiques ont été élaborées qui permettent de diviser la variance d'un caractère quantitatif en composantes inégales dues à des facteurs génétiques - dont l'action peut être de nature additive ou non-additive - et à des facteurs d'environnement. Une planification rationnelle des expériences permet généralement de contrôler 
une part de la variance due à l'environnement. La part restante de cette variance, part qui peut être importante, est appelée variation intangible. On admet généralement qu'elle est due, soit à des facteurs externes de nature inconnue, soit à quelques erreurs ou accidents du développement (FALCONER, I967).

La durée de développement, période comprise entre la ponte de l'œuf et l'émergence de l'imago, est un caractère aisément mesurable. C'est néanmoins un caractère éminemment complexe puisqu'il est le terme final d'un ensemble de processus séquentiels et d'interactions coordonnées. Depuis les travaux de AlPaTov et PEARL (I929), qui ont mis en évidence l'influence de la température sur la durée de développement, il est bien connu que les modifications de l'environnement peuvent l'altérer profondément. L'humidité relative (ELWYN, I9I7), la densité de population préimaginale (SANG, I949 ; LINTS, I963), la photopériodicité (PITTENDRIGH, 1954), la qualité et la quantité de la nourriture (SANG, I956, I959), l'âge parental (LINTS, I960) sont autant de facteurs susceptibles de le modifier à des degrés plus ou moins grands.

Quant à son déterminisme polygénique aucune démonstration définitive, telle celle que MATHER (I94I) a donnée pour le caractère nombre de soies abdominales, n'en a jamais été faite. Cependant nombreuses sont les expériences de sélection effectuées pour ce caractère, ce qui, entre autres choses, signifie que certains expérimentateurs admettent qu'il a un déterminisme de nature polygénique et lui supposent en outre une part importante de variance additive (SANG et CLAYTON, I957; HUNTER, I959; Clarke, Maynard Smith et Sondhi, I96i ; Prout, I962; Sang, ig62; BAKKer, I969). Des expériences basées sur la consanguinité et les croisements entre lignées consanguines laissent entendre que d'autres chercheurs lui supposent une part de variance non-additive.

Un fait marquant propre aux expériences de sélection pour la durée de développement réside en ceci que les résultats sont souvent exprimés en termes d'écarts à un témoin ramené à zéro (Clarke, Maynard Smith et Sondhr, I96I ; Prout, r962). Lorsque les résultats sont exprimés en valeurs réelles, les maigres fluctuations de la durée que les auteurs pensent être dues aux effets de la sélection sont très souvent masquées par des fluctuations beaucoup plus importantes, considérables d'une génération à l'autre, et apparemment incohérentes (HUNTER, I959); ; les auteurs les attribuent alors à des changements inconnus et incontrôlables de l'environnement (SOKAL, et Hunter, I956).

Il est de pratique générale dans les expériences de sélection - et cela est certainement vrai dans les expériences de sélection pour la durée de développement de ne se préoccuper que du caractère pour lequel on sélectionne. Et pourtant les rapports entre durée de développement et taille de l'imago sont extrêmement complexes et ne devraient pas être ignorés dans pareil contexte. Il a ainsi été démontré dans une population sauvage et dans toute une série de conditions expérimentales précises que la taille à l'émergence décroissait lorsque la durée de développement était prolongée ce qui suggère, mais ne démontre pas, que ces caractères sont génétiquement liés (LINTS, I963). Les rapports entre taille à l'émergence et durée de développement dépendent également du milieu; ainsi lorsque la température d'élevage décroit la durée de développement est prolongée (LOEB et NORTHROP, I9I7) et la taille accrue (AlPATOV et PEARL, I929); par contre lorsque la densité de population larvaire est augmentée la taille à l'émergence est diminuée tandis que la durée de développement est prolongée (SANG, I949 ; LINTS, I963 ; LINTS et LINTS, I969 a). Il est impor- 
tant de se rendre compte qu'en fonction des circonstances les relations entre les deux caractères peuvent être entièrement inversées (LiNTS et LiNTS, I965, I969a).

Tenant compte de ces faits, une expérience de sélection deux-voies a été réalisée de manière telle qu'elle permette d'étudier plus avant le déterminisme de la durée de développement. Ainsi tous les facteurs d'environnement susceptibles d'influencer la durée d'une manière ou d'une autre ont été définis et maintenus aussi constants que possible tout au long de l'expérience. De même une forte intensité de sélection pour une durée de développement réduite ou prolongée a été appliquée à la population sous sélection de telle sorte qu'aucune sélection pour ce caractère ne puisse se faire par le biais d'une sélection inconsciente pour la taille imaginale. Enfin une sélection deuxvoies pour la taille - accompagnée de mesures précises pour la durée de développement - a été pratiquée sur la même population, dans les mêmes conditions d'environnement, avec les mêmes précautions expérimentales, mais avec un délai de dix jours par rapport à l'expérience de sélection pour la durée. Ce décalage de dix jours permet d'être certain que de légères modifications de l'environnement ne peuvent influencer de la même manière les durées de développement observées dans les deux expériences de sélection.

Il est montré que la sélection pour la durée de développement, quand elle n'est pas influencée par une sélection pour un autre caractère, est inefficace; que des fluctuations importantes de la durée de développement s'observent de génération en génération; que ces fluctuations ne dépendent ni de l'environnement, ni du génotype, mais sont déterminées, à une génération donnée, par la durée de développement de la génération précédente.

\section{MATÉRIEL E'T MÉTHODES}

La souche utilisée est Cugo (GRUwez et al, r97I), souche "sauvage " artificielle élevée en masse à $25^{\circ} \mathrm{C}$ durant cinq générations et ensuite à $22^{\circ} \mathrm{C}$ durant quatre générations.

Deux expériences de sélection deux-voies sont réalisées : la première pour la taille thoracique, la seconde pour la durée. A fin de ne faire aucune sélection inconsciente pour un caractère par une sélection pour l'autre, les individus n'appartenant pas au mode du caractère non sélectionné soit environ $3^{\circ} \mathrm{p}$. I oo des individus - sont éliminés avant que la sélection d'une intensité $i=\mathrm{I} .23$, ce qui équivaut à retenir $25 \mathrm{p}$. Ioo des individus restants, ne soit effectuée. De même les témoins pour un caractère sont choisis parmi les individus appartenant au mode de l'autre caractère.

Toutes les expériences sont réalisées à $22 \pm 0,5^{\circ} \mathrm{C}$. L'humidité relative est régularisée à ro p. Ioo près. La photopériodicité est de I 2 heures d'obscurité - de 2 I à 9 heures — suivies de I 2 heures de lumière. Les œufs récoltés en vue de produire la génération suivante sont toujours pondus par des femelles âgées de 8 à 9 jours pendant une période de 24 heures, ceci a fin d'éviter l'influence sur la durée de développement du moment de ponte d'une part (BAKKER et NELISSEN, I 962) et du cycle journalier de ponte d'autre part (GRUwez et al., I97 I). Les œufs ainsi récoltés sont redistribués par lots de 20 sur un milieu de culture classique : on connaît les effets de la densité de population larvaire tant sur la taille que sur la durée de développement (LiNTs et LiNTs, I969 a). Les imagos sont récoltés et dénombrés toutes les quatre heures en période diurne, soit de 9 à 2 I heures; par contre les émergences - fort peu nombreuses (PITTENDRIGH, I954; BAKKER et NELISSEN, I962) - de la période nocturne ne sont recensées qu'une fois, soit à 9 heures du matin. Un nombre total d'environ 600 individus, par lignée, est ainsi récolté à chaque génération. Taille et durée de développement de chacun de ces individus sont mesurées.

Un dernier point doit être souligné. Les deux expériences de sélection débutent à partir d'une même génération de la souche Cugo. Lors de la reproduction les deux générations parentales des lignées témoin et de sélection sont d'un âge identique, descendent des mêmes individus mais proviennent de pontes séparées de to jours. La première ponte produit la génération $\mathrm{P}$ des lignées sélectionnées pour la durée de développement, la seconde la génération $\mathrm{P}$ des lignées sélectionnées pour la taille. Les générations successives des deux sélections sont donc continuellement élevées à 
dix jours d'intervalle. Les fluctuations mineures de l'environnement, qui, nous l'avons vu, sont souvent jugées responsables des variations de la durée de développement d'une génération à l'autre, ne peuvent donc jamais affecter les deux expériences de sélection de la même manière.

\section{RÉSULTATS}

Les figures I et 2 montrent ce que sont les variations de la durée de développement des femelles (A) et des mâles (B) dans les lignées contrôle, haute et basse des sélections deux-voies pratiquées pendant huit générations pour la durée de développement (fig. I) et pour la taille (fig. 2).

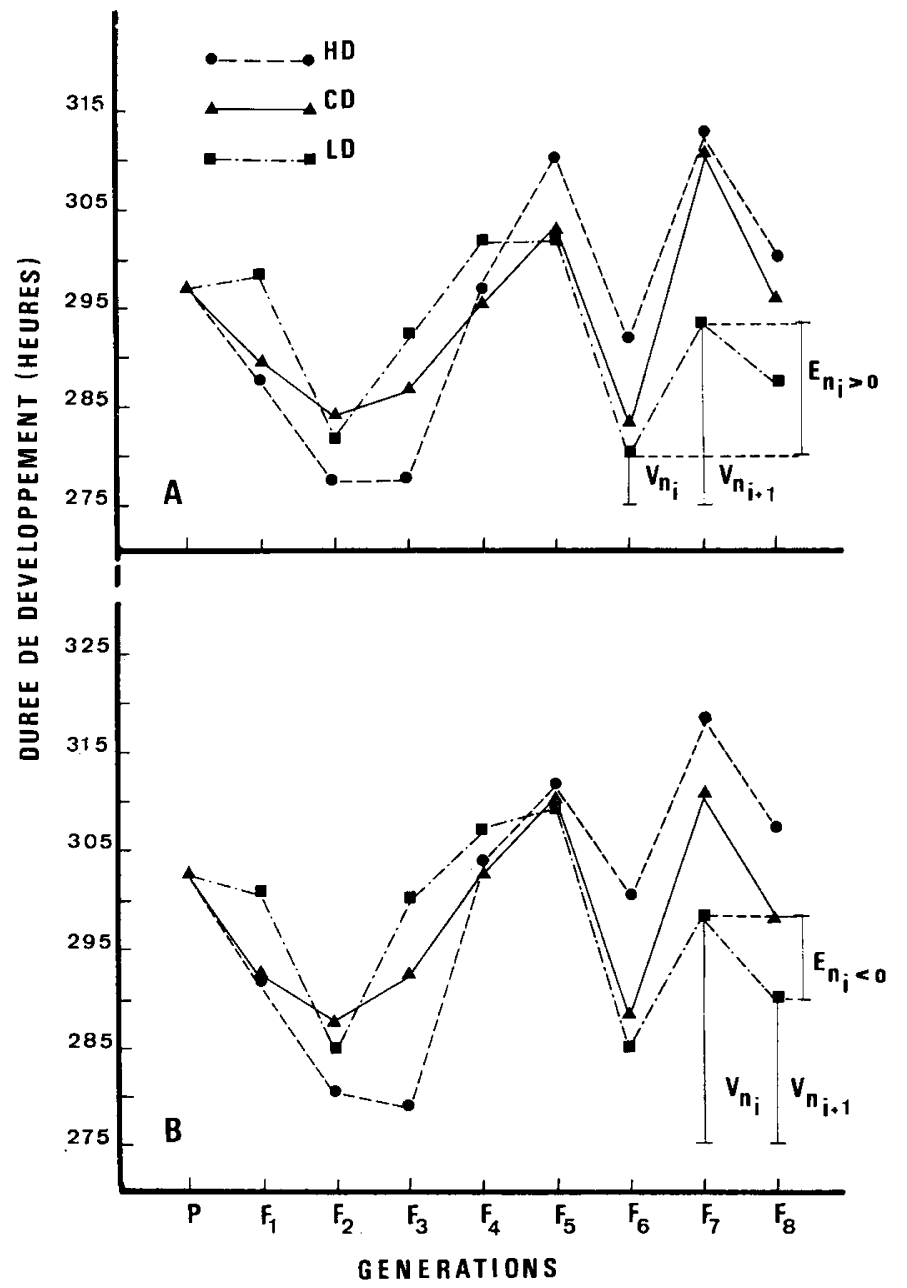

FIG. I. - Variations de la durée de développement observées pendant huit générations d'une sélection deux-voies pour la durée de développement

$$
\text { _- lignée haute; }
$$

A : femelles ; $B$ : mâles.

Les explications concernant $\mathrm{E}_{\boldsymbol{n}_{i}}$ et $\mathrm{V}_{\boldsymbol{n}_{i}}$ sont données dans le texte 
La sélection pour la durée de développement, lorsqu'elle est réalisée à partir d'individus de taille identique, puisque appartenant au mode de la taille, est pratiquement inefficace. Les régressions de la durée de développement sur les écarts de sélection cumulés - soit les héritabilités réalisées - ne sont pas significatives (tabl. I). La sélection pour la taille est par contre tout à fait efficace (tabl. 2).

Les deux groupes de graphiques présentent des similitudes intéressantes. Ainsi les coefficients de corrélation calculés entre les durées de développement des deux

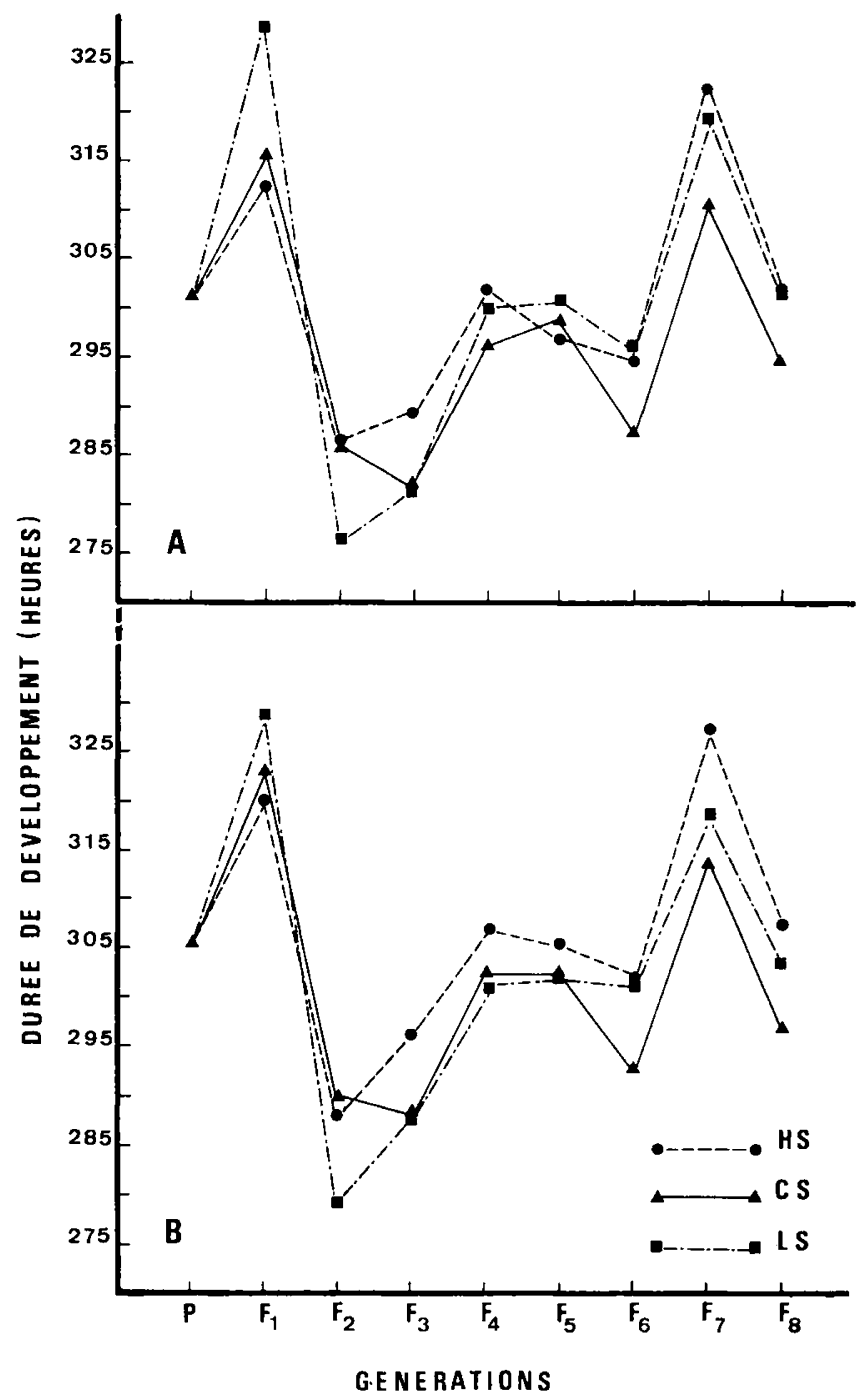

FIG. 2. - Variations de la durée de développement observées pendant huit générations d'une sélection deux-voies pour la taille

-.... lignée haute;

- - - - lignée basse ;

A : femelles ; B : mâles 
témoins - la génération $n$ en sélection taille étant corrélée avec la génération $n$ en sélection durée, $n+\mathrm{I}$ avec $n+\mathrm{I}$ et ainsi de suite - sont très significatifs tant chez les femelles $(r=0,93 ; n=8 ; \mathrm{P}<0,00 \mathrm{I})$ que chez les mâles $(r=0,90 ; n=8$; $\mathrm{P}<0,00 \mathrm{r})$. De plus une analyse de variance appliquée aux données concernant les

\section{TABLEAU I}

Relation entre la durée de développement et l'écart de sélection cumulé (exprimé en heures) pour les lignées sélectionnées

Aucun des coefficients de corrélation et de régression n'atteint le niveau critique de signification de 5 p. Ioo $(n=9)$

\begin{tabular}{|c|c|c|c|c|}
\hline & \multicolumn{2}{|c|}{ Femelles } & \multicolumn{2}{|c|}{ Mâles } \\
\hline & Lignée haute & Lignée basse & Lignée haute & Lignée basse \\
\hline$r$ & $0,548 \mathrm{NS}$ & $-0,267 \mathrm{NS}$ & $0,573 \mathrm{NS}$ & - $0,238 \mathrm{NS}$ \\
\hline$a$ & 284,8 & 295,8 & 288,5 & 300,6 \\
\hline$b$ & 0,079 & $-0,031$ & 0,092 & $-0,029$ \\
\hline$s_{b}$ & 0,0405 & 0,0375 & 0,0439 & 0,0395 \\
\hline$t$ & 1,95 & 0,83 & 2,10 & 0,73 \\
\hline
\end{tabular}

NS : non significatif.

durées de développement des deux expériences démontre que dans les deux cas il n'existe pas de différences significatives entre les lignées témoin, haute et basse (tabl. 3). Ceci permet de grouper les données des trois lignées dans les deux expériences et de

TABLEAU 2

Relation entre la taille et l'écart de sélection cumulé (exprimé en $\mathrm{mm})(n=9)$

\begin{tabular}{|c|c|c|c|c|}
\hline & \multicolumn{2}{|c|}{ Femelles } & \multicolumn{2}{|c|}{ Mâles } \\
\hline & Lignée haute & Lignée basse & Lignée haute & Lignée basse \\
\hline $\begin{array}{c}r \\
a \\
b \\
s_{b} \\
t\end{array}$ & $\begin{array}{l}0,755^{* *} \\
1,059 \\
0,110 \\
0,0370 \\
2,98\end{array}$ & $\begin{aligned}- & 0,884^{* *} \\
& 1,057 \\
- & 0,300 \\
& 0,0465 \\
& 6,46\end{aligned}$ & $\begin{array}{l}0,732^{*} \\
0,940 \\
0,139 \\
0,0395 \\
3,51\end{array}$ & $\begin{aligned}-0,855^{* *} \\
0,934 \\
-0,271 \\
0,0322 \\
8,43\end{aligned}$ \\
\hline & $\begin{array}{l}<P<0,05 \\
1<P<0,01\end{array}$ & & & \\
\hline
\end{tabular}


plus autorise une comparaison entre ces deux expériences basée sur trois fois neuf données ( $\left.{ }^{1}\right)$.

Les coefficients de corrélation entre l'ensemble des données concernant la durée de développement des deux expériences sont significatifs tant pour les femelles $(r=0,680 ; n=24 ; \mathrm{P}<0,00 \mathrm{I} ; b=0,64 \mathrm{I} ; a=$ I03,30) que pour les mâles $(r=0,707$;

\section{TABLEAU 3}

Analyse de variance pour la durée de développement observée pendant huit générations de sélection deux-voies pour la durée de développement et de sélection deux-voies pour la taille

\begin{tabular}{|c|c|c|}
\hline & $\begin{array}{c}\text { Sélection pour } \\
\text { la durée de } \\
\text { développement }\end{array}$ & $\begin{array}{l}\text { Sélection pour } \\
\text { la taille }\end{array}$ \\
\hline Femelles & & \\
\hline $\begin{array}{l}\text { Lignée haute comparée à Lignée témoin... } \\
\text { Lignée haute + Lignée témoin comparées à }\end{array}$ & & $\mathrm{F}_{14}^{\mathbf{1}} 0,95 \mathrm{NS}$ \\
\hline Lignée basse $\ldots \ldots \ldots \ldots \ldots \ldots \ldots \ldots$ & $\mathrm{F}_{25}^{1} 0,16 \mathrm{NS}$ & $\mathrm{F}_{22}^{1} 0,00 \mathrm{NS}$ \\
\hline Mâles & & \\
\hline $\begin{array}{l}\text { Lignée haute comparée à Lignée témoin... } \\
\text { Lignée haute + Lignée témoin comparées à }\end{array}$ & $\mathrm{F}_{16}^{1} 0,03 \mathrm{NS}$ & $\mathrm{F}_{14}^{1} 0,94 \mathrm{NS}$ \\
\hline Lignée basse.................. & $\mathrm{F}_{25}^{1} 0,10 \mathrm{NS}$ & $\mathrm{F}_{22}^{1} 0,13 \mathrm{NS}$ \\
\hline
\end{tabular}

NS : non significatif.

$n=24 ; \mathrm{P}<0,00 \mathrm{I} ; b=0,7 \mathrm{I} 4 ; a=83,46)$. Il devient dès lors évident que les très importantes fluctuations de la durée de développement, qui sont d'un ordre de grandeur de 48 heures et qui sont observées tant en sélection taille qu'en sélection durée, ne peuvent être dues à des causes génétiques, c'est-à-dire à une réponse à la sélection, pour l'excellente raison que dans l'expérience de sélection pour la taille il n'a pas été sélectionné pour le facteur durée. Elles ne peuvent pas non plus être dues à des variations contrôlées de l'environnement pour les raisons qui ont déjà été exposées (Voir Matériel et Méthodes). Elles ne peuvent non plus être dues à des variations intangibles - physiques, chimiques ou même biologiques (SoKAI, et HUNTER, I956) - de l'environnement : le parallélisme presque parfait des données obtenues dans les deux expériences à dix jours d'intervalle rend cette hypothèse insoutenable. Une explication basée sur une contamination virale des souches paraît pour le moins improbable ; on ne voit guère en effet comment les passages de virus d'une génération à l'autre (VoN MAGNus, I95I) et qui se feraient de manière totalement désynchronisée on se souviendra qu'il existe dix jours d'intervalle entre les deux expériences de sélection et l'on notera encore qu'il existe un intervalle de quatre jours entre les individus

(1) La comparaison est en fait réalisée sur trois fois huit données car il a fallu éliminer des calculs la première génération de la sélection taille. Du fait d'une erreur d'ordre technique la densité de population de cette génération est de 40 œufs par tube au lieu de 20 . Cette densité larvaire accrue a provoqué une augmentation de la durée de développement (LinTs et LinTs, I969 a). 
sélectionnés pour un développement rapide et ceux sélectionnés pour un développement lent - pourraient résulter en un parallélisme aussi étroit que celui que nous avons observé dans le comportement de six lignées.

De quoi dépendent alors ces fluctuations?

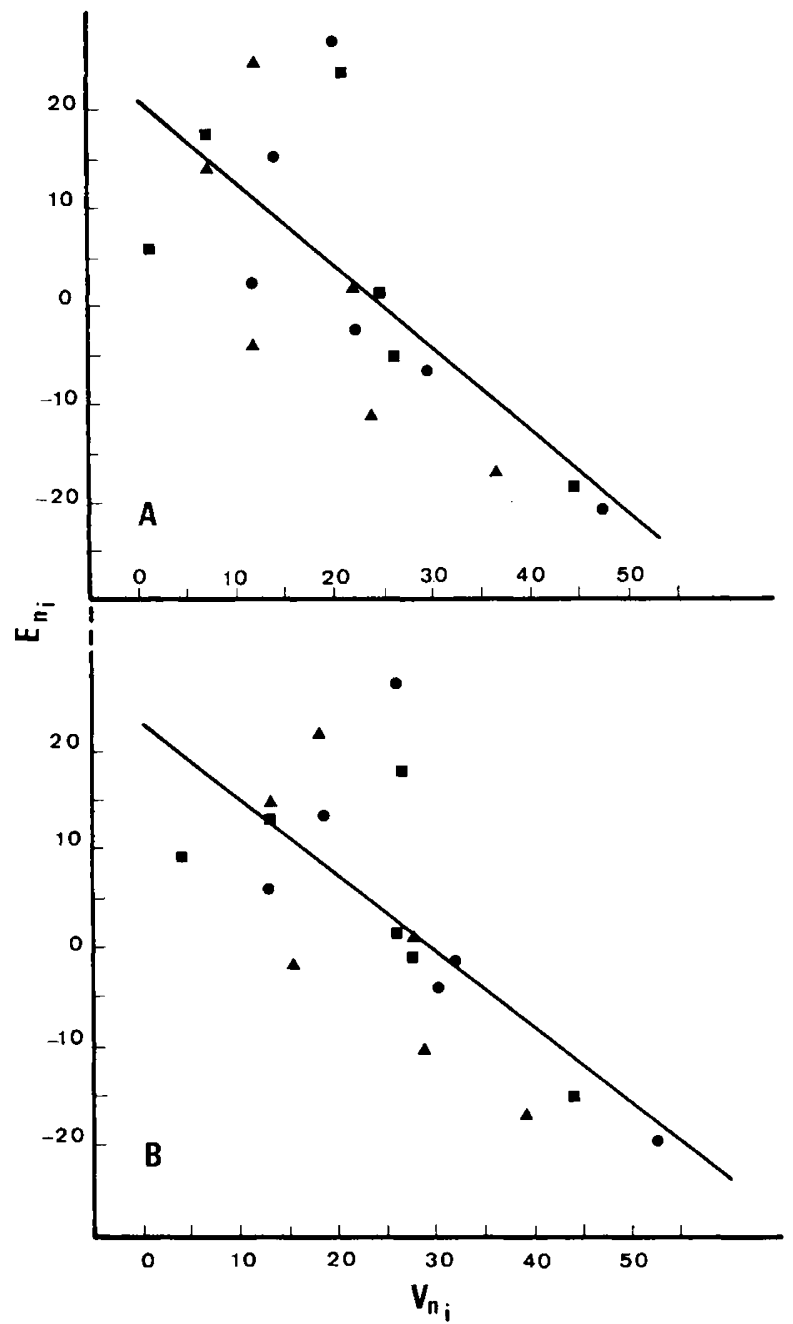

FIG. 3. $-\mathrm{E}_{n_{i}}=f\left(\mathrm{~V}_{n_{\imath}}\right)$ pour les femelles (A) et les mâles (B) des lignées haute $(\bullet)$, basse $(\square)$ et témoin ( $\left.\mathbf{\Delta}\right)$ dans l'expérience de sélection pour la durée de développement

Les durées de développement des générations successives semblent fluctuer entre deux valeurs extrêmes en une sorte d'effet de balance. En d'autres termes il semble exister une relation causale entre la durée de développement de la génération $n$ et celle de la génération $n+\mathrm{I}$. Il ne s'agit pourtant pas d'une relation simple telle celle qui a été postulée par divers auteurs et qui lie une descendance à durée de développement courte à des parents à durée de développement longue, et inversement (LiNTs, I960; DURRANT, 1955). En effet les coefficients de corrélation entre les valeurs succes- 
sives des durées de développement ( $n$ avec $n+\mathrm{I}, n+\mathrm{I}$ avec $n+2$, etc.) sont égaux à $0, \mathrm{I} 42$ pour les femelles ( $n=42$; n.s.) et à 0,223 pour les mâles $(n=42 ;$ n.s.).

Une durée minimale choisie de façon semi-arbitraire et pour des raisons de facilité de calcul a alors été définie : elle est de 275 heures. De fait dans les deux expériences, la durée de développement moyenne la plus faible est de 276,4 heures chez les femelles et de 278,8 heures chez les mâles. A chaque génération et pour chaque lignée de

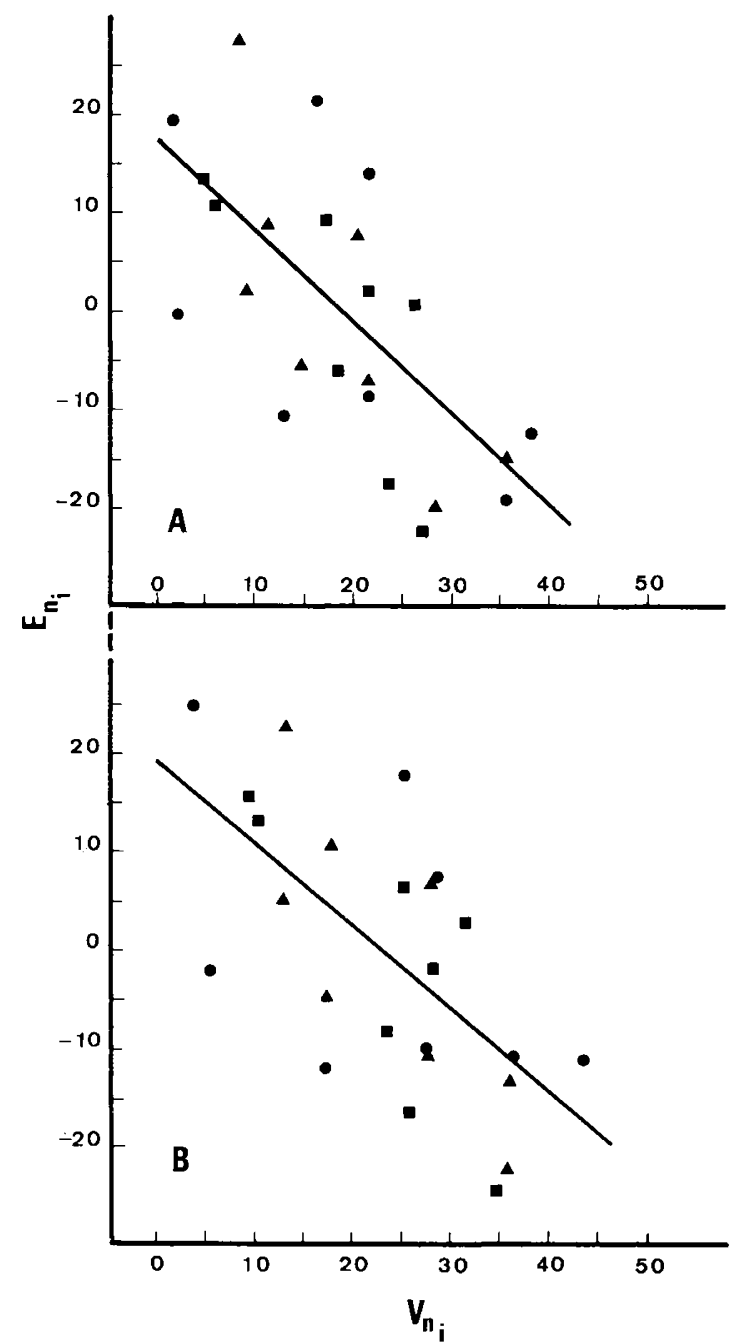

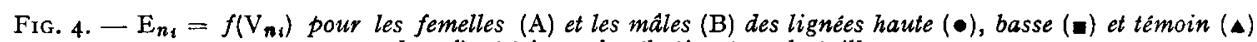
dans l'expérience de sélection pour la taille

sélection les différences entre les valeurs observées et cette limite inférieure ont été calculées : soit à la génération $n_{i}$ la valeur $\mathrm{V}_{n_{i}}$. Ensuite à chaque génération et pour chaque lignée, les différences entre les valeurs successives de $\mathrm{V}_{n_{\mathfrak{i}}}$, soit $\mathrm{E}_{n_{\mathfrak{i}}}$, ont été calculées. $\mathrm{E}_{n_{\mathfrak{t}}}$ peut être positif ou négatif (fig. I). Une régression de la forme $\mathrm{E}_{n_{\mathfrak{l}}}=$ $f\left(\mathrm{~V}_{n_{i}}\right)$ a ensuite été calculée. Autrement dit une génération parentale située au niveau 
$\mathrm{V}_{n_{i}}$ donnera une descendance se situant au niveau $\mathrm{V}_{n_{i}}+\mathrm{E}_{n_{i}}$. Les figures 3 et 4 et le tableau 4 donnent les résultats de ces calculs.

Les corrélations hautement significatives entre $\mathrm{E}_{n_{\imath}}$ et $\mathrm{V}_{n_{\mathfrak{\imath}}}$ démontrent tout d'abord que les variations observées ne sont pas aléatoires ; ensuite que la durée de développement de la génération parentale, définie par rapport à une limite inférieure minimale, affecte de façon considérable la durée de développement de la descendance ; et enfin que c'est la durée de développement d'une génération donnée, exprimée en fonction de la durée de développement minimale, qui détermine à la génération suivante tant l'ampleur que le signe de la déviation par rapport à la génération précédente.

\section{TABLEAU 4}

Relation entre $E_{n_{i}}$ et $V_{n_{i}}$ pour les données regroupées (lignées haute, basse et témoin) des expériences de sélection pour la durée de développement d'une part et de sélection pour la taille d'autre part

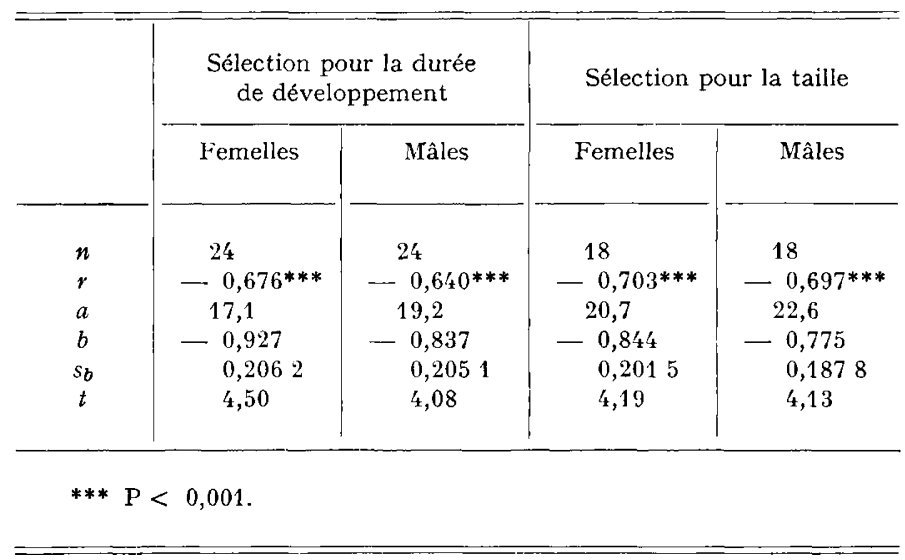

\section{DISCUSSION}

Certes les gènes majeurs peuvent considérablement affecter la durée de développement (LINDSLEY et GREI,L, rg68). Ces gènes néanmoins provoquent en outre des malformations morphologiques et physiologiques importantes et il paraît probable que les modifications de durée de développement sont dans ce cas essentiellement des effets secondaires d'une morphogenèse mal équilibrée.

L'environnement, bien sûr, peut également influencer la durée de développement; à un point tel que plutôt que d'être frappé par la vitesse de croissance de la Drosophile " one of the fastest growing metazoa " (GoRDon, I959) on est bien plus impressionné encore par l'extraordinaire plasticité de son développement.

Par contre l'idée que la durée de développement est un caractère quantitatif polygénique dans le sens classique de cette expression doit être mise en doute. En effet la sélection pour un développement rapide est toujours inefficace (SoKAL et Hunter, I956 ; SANG et Clayton, I957 ; Hunter, I959; Clarke, Maynard Smith 
et SONDHI, I96I ; SANG, I962 ; BAKKER, I969) ; par ailleurs lorsqu'il y a réponse à la sélection pour un développement lent, il n'a jamais été démontré que ce résultat n'était pas dû à une sélection inconsciente pour un autre caractère. De fait dans nos expériences il a été démontré que lorsqu'on sélectionne uniquement et durant 8 générations successives pour la durée la réponse est nulle tant dans un sens que dans 1'autre. Pourtant quand une série de générations successives est mesurée pour la durée de développement, des fluctuations de 1'ordre de 48 heures s'observent d'une génération à 1'autre. SOKAL et HUNTER (I956) qui sélectionnent chez Drosophila melanogaster pour la durée de développement larvaire observent en fait des fluctuations du même ordre de grandeur, de même d'ailleurs qu'un parallélisme étroit entre les lignées haute, basse et témoin. Les fluctuations que nous observons ne sont pas aléatoires : la durée de développement d'une génération donnée dépend de la durée relative de la génération parentale.

La durée de développement est le temps nécessaire à une série de processus séquentiels et d'interactions coordonnées pour donner naissance à un imago plus ou moins bien équilibré. En d'autres termes un imago d'une taille et d'une durée de développement données peut être considéré comme le résultat global d'un ensemble de réactions anaboliques et cataboliques qui, tout en s'opposant, sont plus ou moins bien équilibrées.

Chez Salmo des œufs incubés à basse température produisent des alevins de taille supérieure à ceux produits par des œufs incubés à température plus élevée (GRAY, I929; SPAAs et HEUTS, I958) ; les réserves nutritionnelles de ces œufs étant identiques les variations de taille doivent être dues à une variation dans l'équilibre des processus anaboliques et cataboliques. Da même chez la Drosophile les tailles à l'émergance et les durées de développement sont corrélées positivement ou négativement en fonction des conditions de milieu. Il est dès lors évident que les variations de vitesse de l'ensemble des processus métaboliques - mesurées en première approximation par les variations de la durée de développement - ne peuvent être dues à des variations égales et parallèles de toutes les réactions anaboliques et cataboliques menant à l'imago. Autrement dit, des processus, soit anaboliques, soit cataboliques, peuvent avoir des vitesses complètement différentes en fonction des circonstances : certains constituants cellulaires seront synthétisés, soit en plus grande quantité, soit plus précocement que d'autres dans certaines conditions, tandis qu'en d'autres circonstances ils le seront, soit plus tardivement, soit en quantités moindres. Ainsi chez la Drosophile la vitesse de synthèse de l'ADN est corrélée négativement à la durée de développement mais la teneur absolue en ADN de l'imago est corrélée positivement avec ce même caractère (CHURCH et RoBERTSON, I966). Ainsi encore les quantités relatives des protéines des ribosomes d'Escherichia coli ne sont-elles pas constantes en fonction des conditions d'élevage (Deusser et Wittmann, I972).

La composition et la configuration des divers constituants de la cellule peuvent donc être une fonction des vitesses relatives des processus anaboliques et cataboliques. On connait l'importance en morphogenèse de ce que l'on a appelé la localisation cytoplasmique et que l'on connaît maintenant sous le nom d'information de position (WILsON, I896; CoNkLin, I905; Davidson, I968; LAWRENCE, I97I). Les travaux de GURDON et Brown ( 1965 ) chez Xenopus ont illustré de manière frappante 1'influence du cytoplasme sur le mode d'action génique. Par ailleurs il a été récemment suggéré à partir de résultats expérimentaux que des gradients de divers morphogènes peuvent 
provoquer une croissance différentielle durant l'embryogenèse, autrement dit influencer la dimension spatiale de ce phénomène (CRICK, I970). Les expériences de SoNNEBORN (I970) sur Paramecium ont démontré que les structures ciliaires de cet unicellulaire ont leur orientation déterminée par celle de structures préexistantes; ces structures peuvent être modifiées par micromanipulations et la modification une fois acquise est transmise durant plusieurs générations. Ceci suggère que l'assemblage protéique est déterminé par des assemblages préexistants.

Si les protéines jouent un tel rôle au niveau de la dimension spatiale de la morphogénèse on peut se demander dans quelle mesure elles ne jouent pas aussi un rôle dans ce que nous appellerons la dimension temporelle de ce même phénomène. Autrement dit, la configuration spatiale des cellules influence-t-elle le moment auquel d'autres constituants de ces mêmes ou d'autres cellules seront synthétisés et/ou la vitesse à laquelle ils seront métabolisés? Il ne semble pas impossible qu'une telle configuration spatiale puisse influencer l'ensemble du métabolisme d'un individu en période de croissance, métabolisme mesuré à partir de la durée de développement.

L'influence de la durée de développement parentale sur celle de la descendance pourrait alors être expliquée par l'hypothèse suivante. La composition et la configuration des constituants cellulaires d'une génération donnée - composition et configuration définies par le cours du développement de cette génération — influence la composition et la configuration cellulaire et, par voie de conséquence, les vitesses relatives des processus anaboliques et cataboliques de la génération descendante. De plus les mécanismes homéostatiques de la Drosophile seraient régis de manière telle que des fluctuations de l'ensemble des processus métaboliques ne seraient possibles qu'entre certaines limites. Dès lors qu'une limite supérieure ou inférieure précise est atteinte une réaction de type "feed-back) est enclenchée, d'où l'effet cyclique observé au cours des générations. C'est par l'intermédiaire des œufs que l'information concernant les taux anaboliques et cataboliques doit passer d'une génération à l'autre. On sait en effet que la durée de développement d'individus d'un génotype donné influence le métabolisme de l'état imaginal. Ainsi, par exemple, si les pontes totales d'individus d'un même génotype sont sensiblement égales, le taux de ponte de ces mêmes individus peut-il être considérablement modifié par leur histoire préimaginale et plus précisément par leur durée de développement (LINTS et LiNTS, I969 $a$, I97I $a$, I97 I $b$ ). De même le taux de consommation d'oxygène varie-t-il en fonction de la durée de développement (Lin'ss et LinTs, I969 b). On sait encore que l'âge maternel - et faut-il rappeler que la ponte journalière varie fortement en fonction de 1'âge - influence la dimension des œufs, la durée de développement et la taille alaire de la descendance (DELCOUR et HEUTS, I968).

\section{SUMMARY}

\section{WHAT DETERMINES THE DURATION OF DEVELOPMENT}

\section{IN DROSOPHILA MELANOGASTER}

Two two-way selection experiments, one for size and the other for duration of development, were designed in such a way as to prohibit any unconscious selection for the one trait through an uncontrolled selection for the other. Size and duration were measured in both experiments. All 
environmental factors known to influence duration of development or size in the one or the other respect were defined, controlled and kept constant as far as possible. It is shown that selection for duration of development, when not biased through selection for another trait, is ineffective ; that major fluctuations in duration of development occur from generation to generation; that those fluctuations do not depend on genotype or environment as classically defined, but are, for a given generation, determined by the duration of development of its parental generation. The results are discussed in terms of the time dimension of morphogenesis.

\section{RÉFÉRENCES BIBLIOGRAPHIQUES}

Alpatov W. W., Pearl R., I929. Experimental studies on the duration of life. XII. Influence of temperature during the larval period and adult life on the duration of life of the imago of Drosophila melanogaster. Am. Nat., 63, 37-67.

BAKKeR K., r969. Selection for rate of growth and its influence on competitive ability of larvae of Drosophila melanogaster. Nether. J. Zool., 19, 54I-595.

Bakker K., Nelissen F. X., r962. On the relations between the duration of the larval and pupal period, weight and diurnal rhythm in emergences in Drosophila melanogaster. Entomol. exp. appl., 6, 37-52.

Church R. B., Robertson F. W., I966. Biochemical analysis of genetic differences in the growth of Drosophila. Genet. Res., 7, 383-407.

Clarke J. M., Maynard Smith J., Sondhi K. C., r96r. Asymmetrical response to selection for rate of development in Drosophila subobscura. Genet. Res., 2, 70-81.

Conklin E. G., 1905. The organisation and cell lineage of the ascidian egg. J. Acad. Natur. Sci., 13, 1.

CRICK F., I970. Diffusion in embryogenesis. Nature, 225, 420-422.

Davidson E. H., 1968. Gene Activity in Early Development. Academic Press, New York-London 375 p.

Delcour J., Heuts M. J., 1968. Cyclic variations in wing size related to parental ageing in Drosophila melanogaster. Exp. Geront., 3, 45-53.

DeUSSER E., WrTtManN H. G., 1972. Ribosomal proteins : variation of the protein composition in Escherichia coli ribosomes as function of growth rate. Nature, 238, 269-270.

Durrant A., I955. Extra-nuclear inheritance in Drosophila melanogaster (Abst.) Heredity, 9, 4 I7.

ELWYN A., rgr7. Effect of humidity on duration and on pupal mortality of Drosophila ampilophila LoEw. Bull. Am. Mus. Nat. Hist., 37, 347-353.

FALconer D. S., 1967. Introduction to Quantitative Genetics. Oliver and Boyd, Edinburgh 365 p.

Gordon H. T., r959. Minimal nutritional requirements of the german roach (Blatella germanica). Am. N. Y. Acad. Sci., '77, 290-351.

Gray J., I929. The growth of fisch. III. The effect of temperature on the development of the eggs of Salmo fario. J.exp. Biol., 6, 125-130.

Gruwez G., Hoste C., Lints C. V., Lints F. A., I97r. Oviposition rhythm in Drosophila melanogaster and its alteration by a change in the photoperiodicity. Experientia, 27, 1414-1416.

Gurdon J. B., Brown $\quad$ D. D., 1965. Cytoplasmic regulation of RNA synthesis and nucleolus formation in developing embryos of Xenopus laevis. J. mol. Biol., 12, 27-35.

Hunter P. E., 1959. Selection of Drosophila melanogaster for length of larval period. Z. Vererbungsl., $90,7-28$.

LAwrence P. A., I97I. The organization of the insect segment. Symp. Sac. Exp. Biol., 25, 379-390.

Lindsley D. L., GRell E. H., I968. Genetic Variations of Drosophila melanogaster. Carnegie Institution of Washington Publ. 472 p.

Lints F. A., 1960. Nucleo-cytoplasmic interactions in Drosophila melanogaster. Genetica, 31, 188-239.

Lints F. A., r963. Size in relation to development time and egg-density in Drosophila melanogaster. Nature, 197, I I $28-$ I 130 .

Lints F. A., I97r. Life-span in Drosophila (Critical review). Gerontologia, 17, 33-5 I.

Lints F. A., LinTs C. V., r965. Depression, heterosis and carry-over effects after many generations of inbreeding with Drosophila melanogaster. Genetica, 36, 183-207.

Lints F. A., Lints C. V., I $969 a$. Influence of preimaginal environment on fecundity and ageing in Drosophila melanogaster hybrids. I. Preimaginal population density. Exp. Geront., 4, 23 I-244.

Lints F. A., Lints C. V., I969 b. Respiration in Drosophila. III. Influence of preimaginal environment on respiration and ageing in Drosophila melanogaster. Exp. Geront., 4, $8 \mathrm{r}-94$.

Limts F. A., Lints C. V., r97x a. Influence of preimaginal environment on fecundity and ageing in Drosophila melanogaster hybrids. II. Preimaginal temperature. Exp. geront., 6, 4I7-426.

Lints F. A., Lints C. V., r97x $b$. Influence of preimaginal environment on fecundity and ageing in Drosophila melanogaster hybrids. III. Developmental speed and life-span. Exp. Geront., 6, 427-445. 
Loeb J., Northrop J. H., I9I7. On the influence of food and temperature on the duration of life. J. biol. Chem., 32, го3-121.

MAther K., I94r. Variation and selection of polygenic characters. J. Genet. 41, I59-193.

PitTENDRIGH C. S., I 954. On temperature independence in the clock system controlling emergence time in Drosophila. Proc. Nat. Acad. Sci. U.S.A., 40, ror8-roz9.

Prour T., I962. The effects of stabilizing selection on the time of development in Drosophila melanogaster. Genet. Res., 3, 364-382.

SANG J. H., I949. The ecological determinants of population growth in Drosophila populations. III. Larval and pupal survival. Physiol. Zool., 22, 183-202.

SANG J. H., r956. The quantitative nutritional requirements of Drosophila melanogaster. J.exp. Biol., 33, 45-72.

SANG J. H., 1959. Circumstances affecting the nutritional requirements of Drosophila melanogaster. Ann. N. Y. Acad. Sci., r7, 352-365.

SANG J. H., I 962 . Selection for rate of larval development using Drosophila melanogaster cultured axenically on different diets. Genet. Res., 3, 90-109.

Sang J. H., Clayton G. A., 1957. Selection for larval development time in Drosophila. J. Heredity, 48, $265-270$.

SokAL R. R., Hunter P. E., r956. Environmentally caused fluctuations in quantitative characters of Drosophila. Proc. roth Int. Cong. Entomol., 2, 843-854.

Sonneborn T. M., i97o. Gene action in development. Proc. Roy. Soc. Lond. B., 176, 347-366.

SpaAs J. T., Heuts M. J., I958. Contributions to the comparative physiology and genetics of the European Salmonidae. II. Physiologie et génétique du développement embryonnaire. Hydrobiologia, 12, I-26.

Von Magnus P., I95I. Propagation of PR 8 strain of influenza A virus in chick embryos. II. The formation of "incomplete" virus following inoculation of large doses of seed virus. Acta Path. et Microbiol. Scand., 28, 278-293.

Wruson E. B., I896. On cleavage and mosaic work. Appendix to H. E. Crampton, Jr. " Experimental studies on gastropod development ". Arch. Entwicklungsmech. Organ., 8, 19. 